\title{
Propagação da poaia (Psychotria ipecacuanha) em diferentes substratos e posicionamento das estacas
}

\author{
Maria de Fatima B Coelho' ${ }^{1}$, Vagner A Teixeira²; Rodrigo Aleixo B Azevedo'; Maria Cristina de F e Albu- \\ querque $^{3}$ \\ ${ }^{1}$ Univ. da Integração Internacional da Lusofonia Afro Brasileira, Av. da Abolição, 7, Campos da Liberdade, 62790-000 Redenção-CE; \\ coelhomfstrela@gmail.com, rodrigo.abazevedo@gmail.com; 고stituto Fed. de Ciência e Tecnologia de Mato Grosso, C. Postal 244, \\ 78200-000 Cáceres-MT; vagneraniceto@gmail.com; ${ }^{3}$ UFMT/FAMEV, Av. Fernando Correa da Costa s/n,78060-900 Cuiabá-MT, \\ albuquerquemcfa@gmail.com
}

\section{RESUMO}

A poaia (Psychotria ipecacuanha), Rubiaceae, é importante espécie medicinal, nativa da América, com a maior área de ocorrência no estado de Mato Grosso, principalmente no município de Cáceres. O objetivo neste trabalho foi verificar o efeito de substratos, da posição de estacas de raiz, e de tipos de estacas na propagação de $P$. ipecacuanha. No primeiro experimento foram usadas estacas de raiz com $2 \mathrm{~cm}$ de comprimento no delineamento experimental de blocos ao acaso, no esquema de parcela subdividida 2 × 2 , sendo duas posições (vertical e horizontal) e dois substratos (areia e espuma fenólica), com 15 repetições com 15 estacas cada. No segundo experimento introduzimos estacas basais e apicais da raiz e da parte aérea nos substratos areia, comercial para hortaliças, solo do ambiente natural da espécie e espuma fenólica. O delineamento experimental foi em blocos casualizados no esquema fatorial 4 x 4 , com quatro repetições e cinco estacas por parcela. As variáveis analisadas foram diâmetro, comprimento, número de brotações e número de folhas por brotação. A produção de mudas de $P$. ipecacuanha pode ser feita com estacas de raiz de $2 \mathrm{~cm}$ de comprimento plantadas na posição horizontal ou estacas da parte aérea apical com duas gemas, plantadas na posição vertical e em substratos areia ou solo da área de ocorrência da espécie.

Palavras chave: Rubiaceae, tipos de estaca, mudas.

\begin{abstract}
Propagation of ipecac (Psychotria ipecacuanha) on different substrates and planting position of cuttings

The ipecac (Psychotria ipecacuanha), Rubiaceae, is an important medicinal plant, native to America with the largest area of occurrence in the state of Mato Grosso, Brazil, mainly in the city of Caceres. The aim of this study was to assess the effect of substrates, position of root cuttings and types of cuttings on ipecac propagation. In the first experiment were used cuttings with $2 \mathrm{~cm}$ in length to root, in the experimental design of randomized blocks in split-plot $2 \times 2$ with two positions (vertical and horizontal) and two substrates (sand and phenolic foam) with 15 replications and 15 cuttings in each. In the second experiment we introduced basal and apical cuttings of the root and shoot in sand, commercial substrate for vegetables, soil of the natural environment and phenolic foam. The experimental design was of randomized blocks in factorial scheme $4 \times 4$ with four replications and five cuttings. The analyzed variables were diameter, length, number of shoots and number of leaves per shoot. Seedlings of ipecac can be obtained by root cuttings, $2 \mathrm{~cm}$ in length, placed horizontally or shoot cuttings with two buds placed vertically. The best substrates were sand and soil of the natural environment of the species.
\end{abstract}

Keywords: Rubiaceae, types of cuttings, plantlets.

(Recebido para publicação em 12 de junho de 2012; aceito em 22 de julho de 2013) (Received on June 12, 2012; accepted on July 22, 2013)

\begin{abstract}
$\mathrm{A}^{2}$ poaia ou ipeca (Psychotria ipecacuanha), Rubiaceae, é reconhecida mundialmente como planta medicinal e ocorre no sub-bosque das florestas tropicais úmidas do Brasil, Colômbia, Venezuela, Peru, Equador, Bolívia, Guianas e América Central (Lameira, 2002).

Poaia é conhecida na Europa desde 1762 e suas raízes têm sido obtidas principalmente por extrativismo (Ocampo, 2007). Em meados do século XX foram iniciadas plantações sob coberturas arbóreas do bosque tropical úmido, primeiramente na Nicarágua (Thieblot,
\end{abstract}

1980) e posteriormente na Costa Rica, estabelecendo-se cultivo com raízes de qualidade, dirigido exclusivamente para as demandas do mercado internacional. Por outro lado, o material do Brasil ainda tem origem no extrativismo, principalmente no estado de Mato Grosso, apesar de Addor (1945) já ter relatado este fato. A exploração indiscriminada tem levado à erosão genética da espécie, colocando-a em processo de extinção (Lameira, 2002).

O extrato da raiz de poaia é utilizado como amebicida, emético e expectorante e os principais componentes são alcalói- des isoquinólicos, dos quais a emetina é o mais importante para a indústria farmacêutica (Trease \& Evans, 1988). O Brasil está entre os principais exportadores de poaia (Costa et al., 2000), seguido do Panamá e Costa Rica. As raízes de poaia brasileira apresentam elevado valor farmacológico devido aos altos teores de emetina e cefalina o que confere à planta um poder emético e amebicida (Assis \& Giuliette, 1999).

Entretanto, os aspectos agronômicos têm sido pouco investigados no Brasil, o que faz com que a espécie não esteja incluída em programas de cultivo e 
fomento. Uma referência importante é o trabalho desenvolvido por Lameira (2002), que recomendou a reprodução por fragmentos de raiz de $5 \mathrm{~cm}$ de comprimento na posição horizontal em câmara úmida, posteriormente transferidas para sacolas contendo substrato constituído de terra de solo superficial e esterco de curral curtido na proporção 1:1. As mudas foram então levadas para o local definitivo com sombreamento de $70 \%$ para evitar a incidência direta de radiação solar.

A propagação sexuada não é recomendada em virtude da baixa e demorada germinação que se inicia de 3 a 6 meses após o plantio (Lameira, 2002). Assim, a busca de melhores métodos para a propagação assexuada da espécie constitui uma das tarefas primordiais para o seu cultivo com fins comerciais.

A estaquia é a técnica de propagação vegetativa mais rápida e de mais fácil execução, sendo muito utilizada nas espécies que apresentam maior facilidade para a formação de raízes adventícias. A técnica consiste na multiplicação de plantas usando segmentos caulinares ou radiculares providos de gemas meristemáticas com capacidade para emitir raízes adventícias, comumente denominados estacas (Hartmann et al., 2008).

A propagação vegetativa por estaquia tem sido utilizada de forma eficiente na multiplicação de algumas espécies medicinais, tais como Ginkgo biloba, pariparoba (Pothomorphe umbellata), alecrim pimenta (Lippia sidoides), bamburral (Hyptis suaveolens), hortelã-japonesa (Mentha arvensis), carqueja (Baccharis trimera), atroveran (Ocimum selloi), crajirú (Arrabidaea chica) (Costa et al., 2007; Ferreira \& Gonçalves, 2007; Ramalho et al., 2007; Chagas et al., 2008; Maia et al., 2008; Oliveira et al., 2008; Mattana et al., 2009; Bitencourt et al., 2010), bem como outras plantas medicinais.

Diversos fatores influenciam o sucesso da propagação vegetativa, entre eles, a parte da planta de onde se retira a estaca, a posição da estaca no ramo, o grau de lignificação, a quantidade de reservas e diferenciação dos tecidos, o tipo de substrato, pelas suas características químicas e físicas, a condição fisiológica da planta-mãe, época de coleta, além de outros fatores (Hartmann et al., 2008).

Lourenço et al. (2000) salientaram que o desenvolvimento da muda depende das propriedades físicas, químicas e biológicas do substrato que a sustenta, o que interfere diretamente na qualidade das mudas. Carrijo et al. (2002) recomendaram que um substrato ideal deva possuir, dentre outras características, porosidade acima de $85 \%$, capacidade de aeração entre 10 e $30 \%$ e água facilmente assimilável de 20 a 30\%. No entanto, a escolha do material a ser utilizado depende não só do objetivo a ser alcançado, mas também da disponibilidade local, do custo de aquisição e da experiência do viveirista (Kämpf, 2005).

O plantio de estacas na posição vertical é mais fácil e rápido para quem executa a atividade; entretanto, na bibliografia consultada não foram encontrados trabalhos avaliando a posição de estacas caulinares e radiculares no plantio. Por serem escassos os estudos agronômicos com a poaia de Mato Grosso e baixa a disponibilidade de material propagativo da espécie, é importante avaliar estacas de comprimento reduzido, tanto da parte aérea como da raiz, bem como substratos mais facilmente encontrados na região a fim de que os resultados possam fomentar o cultivo.

Dessa forma, o objetivo neste trabalho foi verificar o efeito dos substratos areia, solo do ambiente natural da espécie, substrato agrícola e espuma fenólica, do posicionamento vertical e horizontal de estaca de raiz e dos tipos de estaca de poaia na propagação da espécie.

\section{MATERIAL E MÉTODOS}

As plantas de poaia foram coletadas em áreas de ocorrência natural da espécie, no município de Cáceres-MT (1546'26"S; 5758'37'O; $146 \mathrm{~m}$ de altitude) em maio de 2006. As plantas foram encaminhadas para casa de vegetação da Escola Agrotécnica Federal de Cáceres, e conservadas em leito de areia de maio a outubro.

A área do experimento foi mantida coberta com tela de sombreamento a $70 \%$. Neste período registraram-se médias de $27^{\circ} \mathrm{C}$ de temperatura diária e de
$76 \%$ de umidade relativa, medidos no interior da casa de vegetação. A irrigação foi feita por microaspersão a cada 12 h durante 30 minutos, durante os cinco meses em que as estacas permaneceram em leito de areia.

O primeiro experimento foi conduzido em duas etapas: uma realizada em casa de vegetação e outra no campo. $\mathrm{Na}$ casa de vegetação foram utilizados os fragmentos de raízes medindo $2 \mathrm{~cm}$ de comprimento obtidos das plantas conservadas em leito de areia. O delineamento experimental utilizado foi em blocos ao acaso com parcela subdividida $2 \times 2$, sendo que os substratos constituíram as parcelas e as sub-parcelas foram constituídas pelas posições das estacas, com 15 repetições, cada uma com 15 estacas. Os substratos foram areia e espuma fenólica, e as posições foram vertical e horizontal. O experimento foi mantido coberto com tela de sombreamento a $70 \%$, conforme recomendado por Lameira (2002). Durante o período experimental de 90 dias foram realizadas irrigações com uso de microaspersão a cada 12 h durante 30 minutos.

$\mathrm{Na}$ segunda etapa as estacas enraizadas no primeiro experimento foram transplantadas para o campo, no mesmo delineamento. Foram feitos montículos de terra a fim de facilitar a aeração para o estabelecimento das mudas, numa área sombreada com árvores de Tamarindus indica. Neste local foi registrada temperatura média diária de $28^{\circ} \mathrm{C}$ e umidade relativa média de $80 \%$. A irrigação das plantas foi feita diariamente com sistema de irrigação localizada utilizando-se mangueira de santeno, com o cuidado para que não houvesse restrição hídrica para as plantas. A avaliação das plantas foi feita aos 180 dias e aos 270 dias após o plantio em casa de vegetação.

O segundo experimento foi realizado em casa telada com tela de sombreamento a $70 \%$ de sombreamento na Faculdade de Agronomia e Medicina Veterinária, na cidade de Cuiabá-MT, de fevereiro a abril de 2008. As plantas para esses trabalhos foram coletadas em janeiro de 2008, no município de Curvelândia-MT (15³3'56'S; 5759'17’'O, $161 \mathrm{~m}$ de altitude), acondicionadas em baldes com solo do ambiente natural, umedecidas e transportadas até a casa telada 
da faculdade.

O delineamento experimental usado foi em blocos ao acaso em esquema fatorial $4 \times 4$, sendo quatro substratos (areia, substrato comercial para mudas de hortaliças à base de casca de pinus, solo do ambiente natural da espécie, espuma fenólica) e quatro tipos de estaca (apical e basal da raiz, apical e basal da parte aérea) com quatro repetições e cinco estacas por parcela.

As estacas de raízes foram retiradas do segmento inicial mais próximo ao início da parte aérea $(\mathrm{RB})$ e mais próxima da região apical da raiz (RA), sendo retiradas duas estacas em cada segmento. As estacas apresentaram duas gemas e foram plantadas na posição horizontal.

As estacas da parte aérea foram retiradas da porção basal (AB) e da região abaixo da inserção de folhas, apical da parte aérea (AA). As estacas apresentaram duas gemas e foram plantadas na posição vertical, enterrando uma gema no substrato a aproximadamente $2 \mathrm{~cm}$ de profundidade.

Nos dois experimentos foram avaliadas a porcentagem de estacas enraizadas, o número de brotações, o comprimento da brotação, o diâmetro da brotação e o número de folhas. Para as medidas de diâmetro e comprimento foram utilizados paquímetro e régua graduada em centímetros, respectivamente. $\mathrm{Na}$ avaliação do diâmetro, o paquímetro foi posicionado na base da emissão da bro- tação e os dados anotados em milímetros e na avaliação do comprimento, a régua foi posicionada na base da emissão de brotação e a medição feita até o início de inserção da folha no ápice da estaca.

Nos dois experimentos foi utilizado o programa SISVAR (Ferreira, 2008) para os testes de normalidade e homogeneidade das variâncias do erro experimental, e posteriormente para a análise de variância e comparação das médias pelo teste Tukey a 5\% de probabilidade. Os dados do número de brotações e número de folhas foram transformados em $(\mathrm{x}+0,5)^{0,5}$ antes da análise de variância.

\section{RESULTADOS E DISCUSSÃO}

No primeiro experimento, aos 90 dias após o plantio (DAP) houve diferença significativa em diâmetro da brotação e número de folhas para o tipo de estaca e, no número de brotações para a posição do plantio da estaca. Aos 180 e 270 DAP houve diferença significativa em diâmetro da brotação e número de folhas para o tipo de estaca e, no número de brotações para tipo e posição do plantio da estaca. A interação não foi significativa para as características avaliadas.

A porcentagem de estacas enraizadas foi alta em todas as repetições, com valores próximos de $98 \%$ e todas as estacas apresentaram emissão de brotação. Aos 90 DAP o diâmetro das brotações foi maior nas estacas colocadas em areia e na posição horizontal (Tabela 1), mantendo-se esse comportamento nas estacas no transplantio no campo (180 e 270 DAT). O comprimento da brotação não foi afetado pelos tratamentos em qualquer das épocas de avaliação. Nas duas posições de plantio o número de brotações da estaca foi maior no substrato areia até 180 DAP e mantendo essa diferença até 270 DAP apenas para a posição horizontal. $\mathrm{O}$ número de folhas na brotação foi maior no substrato areia e na posição horizontal da estaca até 180 DAP e mantendo essa diferença até 270 DAP apenas na areia.

A boa capacidade de enraizamento e brotação de fragmentos de raiz de poaia em casa de vegetação torna viável o início da formação de mudas sob condições controladas, para depois levá-las ao campo, conforme recomenda Lameira (2002) e como foi confirmado no presente estudo.

Como todas as estacas foram transplantadas enraizadas, diferenças nas características da parte aérea são importantes para avaliar o desenvolvimento das mudas no campo. O maior número médio de folhas em mudas que vieram de areia indica que este substrato foi eficiente para a formação das mudas de poaia.

A areia tem baixa capacidade de retenção de água, boa aeração, boa drenagem e alta densidade (Kämpf, 2005),

Tabela 1. Valores médios do diâmetro e comprimento das brotações, número de brotações e número de folhas de $P$. ipecacuanha em diferentes posições de plantio e substratos aos 90, 180 e 270 dias após o plantio (mean values of diameter and shoot length, number of shoots and number of leaves of P. ipecacuanha in different planting positions and substrates at 90, 180 and 270 days after planting). Cáceres, IFCT, 2007.

\begin{tabular}{|c|c|c|c|c|c|c|c|c|c|}
\hline \multirow{2}{*}{$\begin{array}{l}\text { Dias após o } \\
\text { plantio }\end{array}$} & \multirow[t]{2}{*}{ Substrato } & \multicolumn{2}{|c|}{ Diâmetro (mm) } & \multicolumn{2}{|c|}{ Comprimento (cm) } & \multicolumn{2}{|c|}{ Brotações $\left(n^{0}\right)$} & \multicolumn{2}{|c|}{ Folhas $\left(n^{0}\right)$} \\
\hline & & $\mathbf{V}$ & H & $\mathbf{V}$ & H & $\mathbf{V}$ & $\mathbf{H}$ & $\mathbf{V}$ & $\mathbf{H}$ \\
\hline 90 & Areia & $1,0 \mathrm{aA}$ & $1,0 \mathrm{aA}$ & $1,3 \mathrm{aA}$ & $1,3 \mathrm{aA}$ & $1,2 \mathrm{aA}$ & $1,1 \mathrm{aA}$ & $2,8 \mathrm{aB}$ & $3,3 \mathrm{aA}$ \\
\hline Casa vegetação & Espuma & $0,9 \mathrm{aA}$ & $0,8 \mathrm{bA}$ & $1,2 \mathrm{aA}$ & $1,0 \mathrm{aA}$ & $1,0 \mathrm{bA}$ & $0,9 \mathrm{bA}$ & $2,7 \mathrm{aA}$ & $2,4 \mathrm{bA}$ \\
\hline \multicolumn{2}{|l|}{$\mathrm{CV}(\%)$} & \multicolumn{2}{|c|}{18,21} & \multicolumn{2}{|c|}{22,71} & \multicolumn{2}{|c|}{7,60} & \multicolumn{2}{|c|}{14,89} \\
\hline 180 & Areia & $1,0 \mathrm{aA}$ & $1,1 \mathrm{aA}$ & $1,3 \mathrm{aA}$ & $1,3 \mathrm{aA}$ & $1,3 \mathrm{aA}$ & $1,2 \mathrm{aA}$ & $3,7 \mathrm{aB}$ & $4,1 \mathrm{aA}$ \\
\hline Campo & Espuma & $1,0 \mathrm{aA}$ & $0,9 \mathrm{bB}$ & $1,4 \mathrm{aA}$ & $1,5 \mathrm{aA}$ & $1,1 \mathrm{bA}$ & $0,9 \mathrm{bA}$ & $3,6 \mathrm{aA}$ & $3,2 \mathrm{bA}$ \\
\hline \multicolumn{2}{|l|}{ CV $(\%)$} & \multicolumn{2}{|c|}{16,99} & \multicolumn{2}{|c|}{18,13} & \multicolumn{2}{|c|}{6,81} & \multicolumn{2}{|c|}{10,57} \\
\hline 270 & Areia & $1,4 \mathrm{aA}$ & $1,4 \mathrm{aA}$ & $1,5 \mathrm{aA}$ & $1,5 \mathrm{aA}$ & $1,3 \mathrm{aA}$ & $1,2 \mathrm{aA}$ & $2,9 \mathrm{aB}$ & $3,4 \mathrm{aA}$ \\
\hline Campo & Espuma & $1,3 \mathrm{aA}$ & $1,2 \mathrm{bA}$ & $1,6 \mathrm{aA}$ & $1,7 \mathrm{aA}$ & $1,1 \mathrm{aA}$ & $0,9 \mathrm{bA}$ & $2,8 \mathrm{aA}$ & $2,5 \mathrm{bB}$ \\
\hline $\mathrm{CV}(\%)$ & & \multicolumn{2}{|c|}{13,03} & \multicolumn{2}{|c|}{24,29} & \multicolumn{2}{|c|}{6,81} & \multicolumn{2}{|c|}{14,24} \\
\hline
\end{tabular}

Médias seguidas da mesma letra minúscula na coluna e da mesma letra maiúscula na linha não diferem pelo teste Tukey ( $\mathrm{p} \leq 0,05$ ), para cada período. $\mathrm{V}=$ posição vertical, $\mathrm{H}=$ posição horizontal, $\mathrm{CV}=$ coeficiente de variação [means followed by the same lowercase letters in the columns and capital letters in the lines did not differ from each other by Tukey test $(\mathrm{p} \leq 0,05)$, for each period. $\mathrm{V}=$ vertical position, $\mathrm{H}=$ horizontal position, $\mathrm{CV}=$ coefficient of variation]. 
Tabela 2. Médias do diâmetro e comprimento das brotações de estacas, número de brotações e número de folhas de P. ipecacuanha oriundas de fragmentos da parte aérea apical (AA), aérea basal (AB), raiz apical (RA) e raiz basal (RB) nos substratos areia, substrato comercial, solo e espuma [average diameter and length of shoots, number of shoots and number of leaves of cuttings of $P$. ipecacuanha fragments derived from shoot apical (AA), shoot basal $(\mathrm{AB})$, basal root (RB) and apical root (RA) in sand, commercial substrates, soil and foam substrates]. Cuiabá, UFMT, 2008.

\begin{tabular}{|c|c|c|c|c|}
\hline \multirow{2}{*}{ Substratos } & \multicolumn{4}{|c|}{ Tipos de estaca } \\
\hline & $\mathbf{A A}$ & AB & RA & RB \\
\hline & \multicolumn{4}{|c|}{ Diâmetro (mm) } \\
\hline Areia & $1,16 \mathrm{bA}$ & $1,07 \mathrm{cB}$ & $0,87 \mathrm{bB}$ & $1,18 \mathrm{bA}$ \\
\hline Substrato comercial & $1,07 \mathrm{bB}$ & $1,09 \mathrm{cB}$ & $0,88 \mathrm{bB}$ & $1,45 \mathrm{aA}$ \\
\hline Solo & $1,37 \mathrm{aA}$ & $1,33 \mathrm{aA}$ & $0,90 \mathrm{bB}$ & $1,39 \mathrm{aA}$ \\
\hline Espuma & $1,19 \mathrm{bB}$ & $1,27 \mathrm{bAB}$ & $0,95 \mathrm{bB}$ & $1,34 \mathrm{aA}$ \\
\hline \multirow[t]{2}{*}{ CV $(\%)$} & 9,53 & 12,23 & 9,05 & 11,45 \\
\hline & \multicolumn{4}{|c|}{ Comprimento $(\mathrm{cm})$} \\
\hline Areia & $1,33 \mathrm{bA}$ & $1,08 \mathrm{cB}$ & $0,90 \mathrm{aB}$ & $1,11 \mathrm{bB}$ \\
\hline Substrato comercial & $1,05 \mathrm{cAB}$ & $1,09 \mathrm{cAB}$ & $0,85 \mathrm{aB}$ & $1,38 \mathrm{aA}$ \\
\hline Solo & $1,51 \mathrm{aA}$ & $1,65 \mathrm{aA}$ & $0,91 \mathrm{aC}$ & $1,35 \mathrm{aB}$ \\
\hline Espuma & $1,25 \mathrm{bB}$ & $1,38 \mathrm{bA}$ & $1,03 \mathrm{aB}$ & $1,29 \mathrm{abB}$ \\
\hline \multirow[t]{2}{*}{$\mathrm{CV}(\%)$} & 18,69 & 16,87 & 14,87 & 13,25 \\
\hline & \multicolumn{4}{|c|}{ Brotações (nº) } \\
\hline Areia & $1,20 \mathrm{bB}$ & $1,20 \mathrm{cB}$ & $2,12 \mathrm{aA}$ & $1,36 \mathrm{bB}$ \\
\hline Substrato comercial & $1,10 \mathrm{bB}$ & $1,20 \mathrm{cB}$ & $1,97 \mathrm{bB}$ & $2,32 \mathrm{aA}$ \\
\hline Solo & $2,38 \mathrm{aA}$ & $1,71 \mathrm{aA}$ & $1,92 \mathrm{bB}$ & $1,91 \mathrm{abAB}$ \\
\hline Espuma & $1,61 \mathrm{bB}$ & $1,57 \mathrm{bB}$ & $2,23 \mathrm{aA}$ & $2,03 \mathrm{aA}$ \\
\hline \multirow[t]{2}{*}{$\mathrm{CV}(\%)$} & 18,45 & 14,60 & 10,97 & 15,47 \\
\hline & \multicolumn{4}{|c|}{ Folhas $\left(\mathrm{n}^{0}\right)$} \\
\hline Areia & $1,36 \mathrm{cB}$ & $1,18 \mathrm{bB}$ & $3,50 \mathrm{aA}$ & $1,62 \mathrm{bB}$ \\
\hline Substrato comercial & $1,18 \mathrm{cC}$ & $1,36 \mathrm{bC}$ & $2,78 \mathrm{bB}$ & $3,38 \mathrm{aA}$ \\
\hline Solo & $3,07 \mathrm{aA}$ & $2,11 \mathrm{aB}$ & $3,05 \mathrm{aA}$ & $2,20 \mathrm{abB}$ \\
\hline Espuma & $2,00 \mathrm{bAB}$ & $1,98 \mathrm{aB}$ & $3,52 \mathrm{aA}$ & $2,71 \mathrm{abAB}$ \\
\hline CV $(\%)$ & 25,75 & 11,09 & 13,37 & 11,98 \\
\hline
\end{tabular}

Letras minúsculas comparam médias dentro da coluna e letras maiúsculas comparam medias dentro da linha pelo teste Tukey $(p \leq 0,5) \mathrm{CV}=$ coeficiente de variação [lowercase letters compare means within the column and capital letters compare means inside the line by the Tukey test $(\mathrm{p} \leq 0.5)]$.

e estas características podem ter sido responsáveis por mudas de estacas mais vigorosas, que no campo se destacaram. Luz et al. (2007) também verificaram que a areia foi o substrato que proporcionou os melhores resultados quanto à qualidade das raízes e porcentagem de enraizamento de hortênsia (Hydrangea macrophylla).

Durante o mês de julho de 2007 ocorreu um período de sete dias com temperatura abaixo de $25^{\circ} \mathrm{C}$ no local do experimento na fase de campo e foram observados danos nas estacas: diferença significativa para os tipos de estaca e substratos em todas as características, mas a interação entre tipos de estacas e substratos foi significativa apenas para número de brotações e número de folhas. As estacas da parte aérea independente da posição no ramo (apical ou basal) tiveram maior diâmetro e comprimento das brotações no substrato solo (Tabela 2). As estacas obtidas de raiz na posição basal apresentaram maior diâmetro médio das brotações que as estacas obtidas na posição apical da raiz e em relação ao substrato tiveram menor diâmetro em areia.

O número de brotações foi maior nos fragmentos da parte aérea basal $(1,71)$ e apical $(2,38)$ no substrato solo, enquanto os fragmentos de raiz apical tiveram maior número de brotações no substrato areia $(2,12)$ e na espuma $(2,23)$; já as estacas de raiz basal apresentaram maior número de brotações no substrato comercial, solo e espuma. Este mesmo desenvolvimento ocorreu para a característica número de folhas nas brotações (Tabela 2).

A influência de diferentes tipos de estaca (estaca apical e mediana da parte aérea da planta, ponteira e mediana do estolão) de Mentha arvensis, na região Sul do estado de Minas Gerais, foi estudada por Chagas et al. (2008) que verificaram que as estacas apicais da parte aérea apresentaram maior enraizamento e desenvolvimento da parte aérea e baixa porcentagem de mortalidade.

Por outro lado, Maia et al. (2008), em trabalhos realizados com o enraizamento de estacas de Hyptis suaveolens com diferentes tipos de estacas (apical, mediana e basal), verificaram que as estacas medianas apresentaram $100 \%$ de enraizamento e, nas estacas colhidas das partes apical e basal, apresentaram menor enraizamento, mas acima de 50\% (93 e 86\%, respectivamente) e Ramalho et al. (2007) verificaram o maior enraizamento em estacas basais de carqueja (Baccharis trimera).

Estudos de propagação usando estacas de raiz não são frequentes, e as espécies já testadas são arbóreas e não herbáceas como $P$. ipecacuanha. Em Caesalpinia férrea, Santos (2011) verificou que estacas com $18 \mathrm{~cm}$ de comprimento, provenientes de raízes 
de mudas, quando submetidas à dose de $6.000 \mathrm{mg} \mathrm{L}^{-1}$ de AIB apresentavam $85 \%$ de enraizamento e Silva et al. (2011) obtiveram $51,3 \%$ de enraizamento, por meio de estacas oriundas de raízes de Brosimum gaudichaudii com 2 anos de idade em ambiente sombreado. Em goiabeira, Manica et al. (2002) verificaram que a formação de mudas dessa espécie, por meio de estacas de raízes, é obtida com facilidade e sempre resulta em elevada porcentagem de pegamento, quando são escolhidas aquelas com 0,7 a 1,2 cm de diâmetro e 15 a $22 \mathrm{~cm}$ de comprimento.

Com relação ao tamanho da estaca, Lameira (2002) relatou que na produção de mudas de poaia devem ser usados fragmentos da raiz primária com $5 \mathrm{~cm}$ de comprimento. Em estudos de micropropagação, Reis et al. (2004) avaliaram o efeito do tamanho e posição de explantes de poaia na multiplicação in vitro, com tamanhos de 0,$5 ; 1,0$ e $1,5 \mathrm{~cm}$ nas posições basal, mediana e apical e concluíram que os tamanhos de 1,0 cm e 1,5 $\mathrm{cm}$ dos explantes apresentaram maior número de brotações que os segmentos com $0,5 \mathrm{~cm}$. No presente trabalho foram obtidas mudas com estacas de raiz de $2 \mathrm{~cm}$ e isto representa economia de material, de espaço e de substratos para a propagação. A utilização de areia ou solo como substrato é vantajosa, pois são de baixo custo, de fácil disponibilidade e apresentam boas características de drenagem.

A produção de mudas de poaia pode ser realizada por estacas de raiz de $2 \mathrm{~cm}$ de comprimento plantadas na posição horizontal ou estacas da parte aérea apical com duas gemas, plantadas na posição vertical e em substratos areia ou solo da área de ocorrência da espécie.

\section{AGRADECIMENTOS}

Ao Conselho Nacional de Desenvolvimento Científico e Tecnológico (CNPq) pela concessão de Bolsa de Produtividade à primeira e última autora.

\section{REFERÊNCIAS}

ADDOR AA. 1945. Considerações acerca da ipeca. Rio de Janeiro: Ministério da Agricultura, Serviço de Informação Agrícola, $29 \mathrm{p}$.

ASSIS MC. 1992. Aspectos taxonômicos, anatômicos e econômicos da "ipeca" Psychotria ipecacuanha (Brot.) Stokes. São Paulo: Universidade de São Paulo. 132p. (Dissertação mestrado).

ASSIS MC; GIULIETTIAM. 1999. Morphological and anatomical differentiation in: populations of "ipecacuanha" - Psychotria ipecacuanha (Brot.) Stokes (Rubiaceae). Revista Brasileira de Botânica 22: 205-216.

BITENCOURT J; ZUFFELLATO-RIBAS KC; KOEHLER HS. 2010. Estaquia de Ginkgo biloba L. utilizando três substratos. Revista Brasileira de Plantas Medicinais, 12: 135-140.

CARRIJO AO; LIZ RS; MAKISHIMA N. 2002. Fibra de casca de coco verde como substrato agrícola. Horticultura Brasileira 20: 533-535.

CHAGAS JH; PINTO JEBP; BERTOLUCCI SKV; NALON FH. 2008. Produção de mudas de hortelã-japonesa em função da idade e de diferentes tipos de estaca. Ciência Rural 38: 2157-2163.

COSTA LCB; PINTO JEBP; BERTOLUCCI SKV. 2007. Comprimento da estaca e tipo de substrato na propagação vegetativa de atroveran. Ciência Rural 37: 1157-1160.

COSTA MP; PINTO JEB; FRANÇA SC; LAMEIRA AO; CONCEIÇÃO HO; SANTIAGO EJA. 2000. Crescimento e teor de emetina em plantas de ipeca (Cephaelis ipecacuanha A. Richard.) obtidas in vitro e submetidas às condições de soluções nutritivas em casa de vegetação. Revista Ciência e Agrotecnologia 24: 46-53.

FERREIRA DF. 2008. SISVAR: um programa para análises e ensino de estatística. Revista Symposium 6: 36-41.

FERREIRA MGR; GONÇALVES EP. 2007. Estaquia e crescimento inicial de Crajiru (Arrabidaea chica (Humb. \& Bonpl.) B. Verl.). Revista Brasileira de Biociências 5: 363-365.

HARTMANN HT; KESTER DE; DAVIES JR; FT; GENEVE RL. 2008. Plant propagation: principles and practices. New Jersey: PrenticeHall, 770p.

KÄMPF AN. 2005. Produção comercial de plantas ornamentais. Guaíba: Agropecuária. 254p.

LAMEIRA AO. 2002. Cultivo da ipecacuanha [Psychotria ipecacuanha (Brot.) Stokes]. EMBRAPA, Circular Técnica, 28: 1-4.

LOURENÇO RS; MEDRADO MJS; FOWLER JAP; MOSELE SH. 2000. Influência do substrato no desenvolvimento de mudas de erva-mate (Ilex paraguariensis St. Hill.). Boletim de Pesquisa Florestal 38: 13-30.

LUZ PB; PAIVA PDO; LANDGRAF PRC.
2007. Influencia de diversos tipos de estacas e de substratos na propagação assexuada de hortênsia [Hydrangea macrophylla (Thunb.) Ser.] Ciência Agrotecnica 31: 699-703.

MAIA SSS; PINTO JEBP; SILVAFN; OLIVEIRA C. 2008. Enraizamento de Hyptis suaveolens (L.) Poit. (Lamiaceae) em função da posição da estaca no ramo. Revista Brasileira de Ciências Agrárias 3: 317-320.

MANICA I; ICUMA IM; JUNQUEIRA NTV; SALVADOR JO; MOREIRA A; MALAVOLTA E. 2002. Fruticultura Tropical 6 - Goiaba. Porto Alegre: Cinco Continentes Editora. 374p.

MATTANA RS; FRANCO VF; YAMAKI HO; MAIA; ALMEIDA CI; MING LC. 2009. Propagação vegetativa de plantas de pariparoba [Pothomorphe umbellata (L.) Miq.] em diferentes substratos e número de nós das estacas. Revista Brasileira de Plantas Medicinais 11: 325-329.

OCAMPO R. 2007. Ipecacuana. Un producto no maderable cultivado bajo el bosque en Costa Rica. 1980-2000. Disponível em: http:// www.allbusiness.com/pharmaceuticalsbiotechnology/pharmaceuticalsindustry/5493716-1.html. Acessado em 12 de janeiro de 2012.

OLIVEIRA GL; FIGUEIREDO LS; MARTINS ER; COSTA CA. 2008. Enraizamento de estacas de Lippia sidoides Cham. utilizando diferentes tipos de estacas, substratos e concentrações do ácido indolbutírico. Revista Brasileira de Plantas Medicinais 10: 12-17.

RAMALHO RIN; NOLASCO MA; CARVALHO T; RIPKA M; GIUBLIN LM; NEGRELLO N; SCHEFFER MC. 2007. Enraizamento de estacas de carqueja em função de diferentes substratos e posições do ramo em plantas masculinas e femininas. Scientia Agrária 8: 269-274.

REIS ES; PINTO JEBP; CORRÊA RM; BERTOLUCCI SKV; LAMEIRA AO. 2004. Tamanhos e posições de explantes e volumes de meio de cultivo na multiplicação de ipeca (Psychotria ipecacuanha (Brot.) Stokes) in vitro. Ciência Agrotecnica 28: 703-709.

SANTOS LW. 2011. Propagação e produção de mudas de mulungu, Erythrina velutina Willd. (Fabaceae) e Jucá, Caesalpinia ferrea Mart. ex Tul. (Caesalpiniaceae) em Mossoró-RN. Mossoró:UFERSA 133p. (Tese doutorado).

SILVA DB; VIEIRA RF; CORDEIRO MCT; PEREIRA EBC; PEREIRA AV. 2011. Propagação vegetativa de Brosimum gaudichaudii Tréc. (mama-cadela) por estacas de raízes. Revista Brasileira de Plantas Medicinais 13: 151-156.

THIEBLOT MJ. 1980. A mata da poaia e os poaieiros do Mato Grosso. São Paulo: Escola de Folclore/Editorial Livramento, 80p.

TREASE GE; EVANS WC. 1988. Tratado de farmacognosia. México: Nueva Editorial Interamericana. p.611-615. 